\title{
DYSLIPIDEMIA AND ANTI-OXIDATIVE SYSTEM IN PREECLAMPTIC WOMEN AT RURAL AREA OF RAYALASEMA
}

\author{
Anita Ramesh Annaldasula *1, K Durgaprasad 2. \\ ${ }^{* 1}$ Associate professor, obstetrics \& Gynecology, Shanthiram Medical College, Nandyal, Kurnool, \\ Andhra Pradesh, India. \\ ${ }^{2}$ Professor and head, Biochemistry, Shanthiram Medical College, Nandyal, Kurnool, Andhara Pardesh, \\ India.
}

\section{ABSTRACT}

Oxidative stress increases during pre-eclampsia and results in increased production of lipid peroxides, reactive oxygen species and superoxide anion radicals to cause endothelial injury and dysfunction, platelet and neutrophil activation. In preeclampsia serum antioxidants are excessively utilized to counteract the cellular changes mediated by free radicals. Antioxidants, such as caroteinoids, tocopherols, and ascorbic acid, due to their capacity for scavenging free radicals and their function as inhibitors of reactive oxygen species, are lower in women with preeclampsia with this background the role of oxidative stress in the pathogenesis of preeclampsia in south Indian women. For this reason, to strengthen the existing literature, our primary objective is to assess the status of lipid peroxidation marker MDA and antioxidants such as Vitamin$\mathrm{C}$ and uric acid in women with preeclampsia. This case control study was conducted at Obstetrics and Gynecology department with collaboration with Biochemistry department. 40 controls and 40 cases (preeclamptic women) were included in the pregnant who were attending regular checkup in the Obstructs and Gynecology department op. Oxidative and anti-oxidative stress in cases compared with the controls. The $p$-values were showing the significance difference between the normal pregnant and preeclamptic pregnant and diagrammatically represented. The imbalance between oxidative damage and antioxidant defences in pre-eclampsia leads to endothelial cell dysfunction and it appears to be a central feature in the patho-physiology of pre-eclampsia KEY WORDS: Preeclampsia, MDA, Uric Acid, Vit-C, Oxidative Stress, Antioxidants.

Address for correspondence: Dr. Anita Ramesh Annaldasula, Associate professor, obstetrics \& Gynecology, Shanthiram Medical College, Nandyal, Kurnool, Andhra Pradesh, India.

E-Mail: anitasreekanth123@gmail.com

\begin{tabular}{|l|lc|}
\hline \multicolumn{3}{|c|}{ Online Access and Article Informtaion } \\
\hline Quick Response code & \multicolumn{1}{|c|}{ International Journal of Integrative Medical Sciences } \\
& \multicolumn{1}{c}{ www.imedsciences.com } \\
\cline { 2 - 3 } & Received: 11-10-2017 & Accepted: 20-10-2017 \\
Dol: 10.16965/ijims.2017.114 & Reviewed: 11-10-2017 & Published: 03-11-2017 \\
\hline Source of Funding: Self & \multicolumn{2}{c|}{ Conflicts of interest: None } \\
\hline
\end{tabular}

\section{BACKGROUND}

Preeclampsia(Pregnancy induced Hypertension) is a pregnancy specific condition characterized by hypertension $(140 / 90 \mathrm{~mm} \mathrm{Hg}$ ) and proteinuria (300mg/24hrs) that remits after delivery [1]. Preeclampsia (PIH) affects 3 to $7 \%$ of pregnant women. It is also a major cause of maternal mortality (15\%-20\%) in developed countries and a leading cause of preterm birth and intrauterine growth retardation $[2,3]$.
Preeclampsia may develop from 20 weeks gestation with the etiology is unknown; however, risk factors include the Null parity, Pre-existing chronic hypertension, Vascular disorders (eg, renal disorders, diabetic vasculopathy), Pregestational or gestational diabetes, Older (> $35)$ or very young $(e g,<17)$ maternal age, Family history of preeclampsia, Preeclampsia or poor outcome in previous pregnancies, Multifetal pregnancy, Obesity, Thrombotic disorders like antiphosp-holipid antibody syndrome. 
Lipid peroxidation is a normal phenomenon that occurs continuously at low levels in every individual. These peroxidation reactions are toxic to cells and cell membranes. Following lipid peroxidation aldehyde products, such as free fatty acids, malondialdehyde (MDA), occur and these products are referred to as Thiobarbituric acid reacting substances (TBARS). Since the cell membranes consist primarily of lipids, uncontrolled lipid peroxidation can cause cell injury and death via DNA damage and directly inhibiting proteins [4]. Antioxidants are the substances which will significantly delay or inhibit oxidation of an oxidisable substrate [5] .They act as defense mechanisms against the free radicals.

In preeclampsia the serum lipids show a shift towards a dyslipidemic profile. The serum Triglycerides (TG) and Total Cholesterol (TC) are further elevated, there is a higher proportion of Low density Lipoprotein (LDL) of small size, High density lipoprotein (HDL) decreased and serum free fatty acids (FFA) are increased [6]. Lipid peroxidation and antioxidant status has been implicated in the etiology of preeclampsia [7]. Many reports suggest that lipid peroxidation products, primarily measured as thiobarbituric acid reactive substances (TBARS) which include Malondialdehyde (MDA) are increased in plasma / sera of women with preeclampsia [8].

Oxidative stress increases during pre-eclampsia and results in increased production of lipid peroxides, reactive oxygen species and superoxide anion radicals to cause endothelial injury and dysfunction, platelet and neutrophil activation [9].

In preeclampsia serum antioxidants are excessively utilized to counteract the cellular changes mediated by free radicals. Antioxidants, such as caroteinoids, tocopherols, and ascorbic acid, due to their capacity for scavenging free radicals and their function as inhibitors of reactive oxygen species, are lower in women with preeclampsia [10]. MDA is the end product of lipid peroxidation and reflects the oxidative status of the biological system [11].

Caffeine and uric acid are good antioxidants. Increased uric acid level is a key clinical feature of preeclampsia; higher levels correlate with significant maternal and fetal morbidity and Int J Intg Med Sci 2017;4(6):517-21. ISSN 2394 - 4137 mortality. The cause of hyperuricemia and its specific role in the pathogenesis of preeclampsia, however, remain unclear. Although uric acid has been shown to roughly parallel the severity of the maternal syndrome, it has not been useful in predicting the development of preeclampsia. Nevertheless, there have been recent data supporting a pathogenic role potentially in the hypertension and endothelial cell dysfunction of preeclampsia [12].

Vitamin $C$ is the important aqueaus phase antioxidant. Current public health efforts to increase intake of fruits and vegetables rich in vitamin $\mathrm{C}$ and other antioxidants may reduce the risk of preeclampsia [13]. These observations in pre-eclampsia have given rise to increased interest in antioxidants such as Ascorbic acid (Vitamin C) and Uric acid.

The findings of the previous studies, suggested that oxidative stress through increased lipid peroxidation is an important factor in the pathogenesis of preeclampsia. As far as our knowledge is concerned, till date there are only few reports of oxidative stress and antioxidant status in preeclampsia from India. Recently, Kashinakunti SV et al [14] and Sharma JB et al., [15] reported the role of oxidative stress in the pathogenesis of preeclampsia in Indian women. For this reason, to strengthen the existing literature, our primary objective is to assess the status of lipid peroxidation marker MDA and antioxidants such as Vitamin-C and uric acid in women with preeclampsia.

\section{MATERIALS AND METHODS}

\section{Study design:}

This case control study was conducted.

This study was conducted at Obstetrics and Gynecology department with collaboration with Biochemistry department. We included in the 80 pregnant who were attending regular checkup in the Obstetrics and Gynecology department op at Shanthiram Medical college, Nandyal, Kurnool, AP. divided in to control group (40 normal pregnant women) and cases (40 preeclamptic women).

\section{Inclusion criteria:}

Women were included in the study if they lived 25 kilometers or less from the hospital, Both the 
cases and controls will be between 18 and 30 years with more than 29 weeks of gestation, and were willing to deliver at the hospital.

\section{Exclusion criteria:}

Individuals having past history of Diabetes mellitus, Hypertension, renal diseases, liver disorder, cardiac diseases or sickle cell disease and with the history of multivitamin intake and drugs that affect lipid status were excluded.

\section{Data collection:}

The women were interviewed about their sociodemographic characteristics, medical and family history, and the present and past obstetric performances. In the socio demographic characteristics information was obtained about the age of the mother in completed years, the marital status, the level of education, and information of the mothers' socio economic status.

The women examined had a general and obstetric examination and, the blood pressure and proteinuria were taken. Fasting Blood was drawn the serum will be separated and analyzed for the following parameters at the time of recruitment for lipid profile, uric acid, MDA, and vitamin $C$ assays.

\section{Sample analysis:}

MDA will be measured by Thiobarbituric acid reactive substances assay (TBRAS) method [16] and ascorbic acid by Dinitrophenyl Hydrazine ( DNPH) method [17]. The uric acid will be measured by Uricase method using Human diagnostics kit [18].

The optical densities of MDA and ascorbic acid will be measured at $532 \mathrm{~nm}$ and $520 \mathrm{~nm}$ respectively using spectrophotometer. Serum lipid profile (TG, TC, HDL-C) by enzymatic colorimetric methods [19].

Serum LDL $-C$ and VLDL will be calculated using Friedwald's formula: LDL-C $=$ TC-(TG/5 + HDL-C) and $\mathrm{VLDL}=\mathrm{TG} / 5$.

For vitamin C assays, fresh blood was centrifuged within four hours to separate the plasma. The plasma was kept at $-80^{\circ} \mathrm{C}$ until analysis. Vitamin $\mathrm{C}$ was assayed using a colorimetric method as described by Kyaw24. This method determines ascorbic acid using acid phosphotungastate. Briefly, 100il of color reagent (acid phosphotungastate) was added slowly to 100il of plasma in centrifuge tubes. The solution was mixed with a vortex mixer and was allowed to stand for 30 minutes at room temperature. It was centrifuged at $3000 \mathrm{rpm}$ for 15 minutes. The blue-colored supernatant that formed was transferred to the Elisa plate Multiscan Ex (Elisa Reader) Serial No: RS-232. The multiscan Ex is an eight channel light path filter photometer designed to perform standard photometric measurements. The absorbance at $620 \mathrm{~nm}$ was read in pairs against a blank which was constituted with distilled water.

\section{Statistical analysis:}

All values obtained will be expressed as Mean ( \pm SEM). Unpaired two-tailed student $t$-test will be performed to compare the difference in the means between controls and study group. A ' $P$ ' value $<0.05$ will be considered as statistically significant. Statistical Analysis will be done by using Microsoft excel spread sheets.

\section{RESULTS}

Table 1: Showing the mean and SD of hypertension and proteinuria in cases compaired with the controls. The $p$-values were showing the significance difference between the normal pregnant and preeclamptic pregnant.

\begin{tabular}{|c|c|c|c|c|}
\hline & & Mean & SD & p-Value \\
\hline \multirow{2}{*}{ SBP } & cases & 137.2 & 35.83 & \multirow{2}{*}{0} \\
\cline { 2 - 5 } & control & 102.5 & 6.35 & \\
\hline \multirow{2}{*}{ DBP } & cases & 77.7 & 10.67 & \multirow{2}{*}{0} \\
\cline { 2 - 4 } & control & 67.75 & 5.77 & \\
\hline \multirow{2}{*}{ PROTEINURIA } & cases & 372.03 & 19.18 & \multirow{2}{*}{0} \\
\cline { 2 - 4 } & control & 153.78 & 4.22 & \\
\hline
\end{tabular}

Table 2: Showing the mean and SD of lipid profile in cases compared with the controls. The $p$-values were showing the significance difference between the normal pregnant and preeclamptic pregnant.

\begin{tabular}{|c|c|c|c|c|}
\hline & & Mean & SD & p-Value \\
\hline \multirow{2}{*}{ CHOL } & cases & 261.28 & 22.62 & \multirow{2}{*}{0} \\
\cline { 2 - 4 } & control & 160.73 & 12.28 & \\
\hline \multirow{2}{*}{ TGL } & cases & 180.45 & 15.21 & \multirow{2}{*}{0} \\
\cline { 2 - 4 } & control & 124.35 & 16.53 & \\
\hline \multirow{2}{*}{ HDL } & cases & 19.9 & 4.51 & \multirow{2}{*}{0} \\
\cline { 2 - 4 } & control & 38.7 & 3.16 & \\
\hline \multirow{2}{*}{ LDL } & cases & 205.29 & 22.69 & \multirow{2}{*}{0} \\
\cline { 2 - 4 } & control & 97.16 & 15.69 & \\
\hline \multirow{2}{*}{ VLDL } & cases & 36.09 & 3.04 & \multirow{2}{*}{0} \\
\cline { 2 - 4 } & control & 24.87 & 3.31 & \\
\hline
\end{tabular}


Table 3: Showing the means and SD of oxidative and anti-oxidative stress in cases compared with the controls. The p-values were showing the significance difference between the normal pregnant and preeclamptic pregnant and diagrammatically represented.

\begin{tabular}{|c|c|c|c|c|}
\hline & & Mean & SD & p-Value \\
\hline \multirow{2}{*}{ MDA } & cases & 2.45 & 0.36 & \multirow{2}{*}{0} \\
\cline { 2 - 4 } & control & 0.91 & 0.26 & \\
\hline \multirow{2}{*}{ URIC ACID } & cases & 8.15 & 0.9 & \multirow{2}{*}{0} \\
\cline { 2 - 4 } & control & 4.61 & 0.64 & \\
\hline \multirow{2}{*}{ VIT-C } & cases & 1.67 & 0.37 & \multirow{2}{*}{0} \\
\cline { 2 - 4 } & control & 3.58 & 0.35 & \\
\hline
\end{tabular}

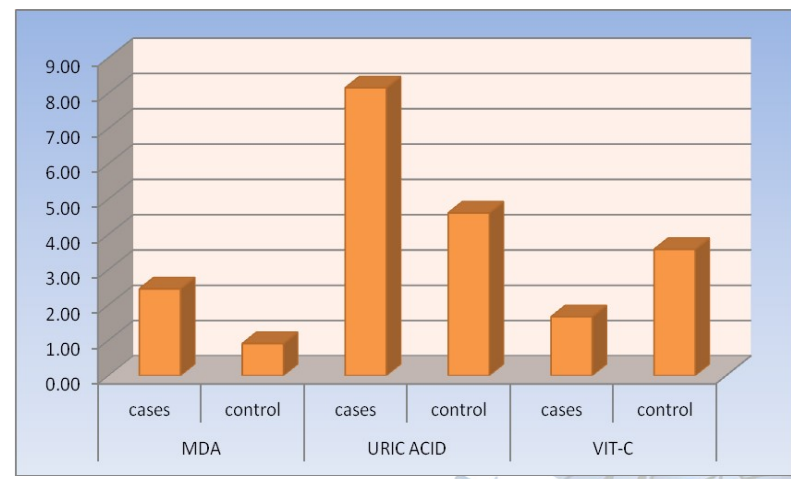

\section{DISCUSSION}

Pathophysiology of pre-eclampsia is poorly understood, it is a pregnancy specific condition characterized by hyper tension and proteinuria, it may develops from 20 weeks gestation with the etiology is unknown. In the present study we observed significant differences in systolic and diastolic blood pressure $(p=0.00)$ as well as urinary protein-they were higher in preeclamptic pregnant women than in the control groups $(p=0.00)$, and this is expected as regards the basis of diagnosing pre-eclampsia. Increase in oxidative stress markers has been implicated to damage the maternal vascular endothelium leading to the elevation in diastolic pressure which further aggravates the condition of preeclamptic patients $[18,19]$.

The imbalance between oxidative damage and antioxidant defences in pre-eclampsia leads to endothelial cell dysfunction and it appears to be a central feature in the patho-physiology of preeclampsia [17]. Compared with healthy pregnant women, preeclamptic women have low levels of several dietary antioxidants in their blood, including vitamin C, vitamin E, and lycopene and beta carotene. They also have higher levels of Reactive Oxygen spices (ROS) and frequently have increased levels of uric acid, probably resulting from the body's attempt to cope with oxidative stress. Production of radicals is directly involved in the oxidative destruction of macromolecules such as lipids, proteins and nucleic acid [16]. Free radicals and other damaging reactive oxygen species can cause lipid peroxidation by oxidative metabolic processes then their activation is thought to increase during pre-eclampsia. In the course of the reaction of free radical with polyunsaturated membrane lipid, a systemic stable product malondialdehyde is produced and this is currently being used as an index of free radical injury. In our study, we observed an increase in plasma malondialdehyde level in preeclamptic women when compared with normal pregnant our findings is in agreement with similar studies which reported a significantly elevated level of malondialdehyde in both normal and preeclamptic pregnant women $[17,18]$.

Endothelial cell dysfunction in glomerula leads to reduction in renal perfusion and glomerular filtration in pre eclampsia. While this condition, developing of hyper uricemia by impairment of tubular function and reduction in uric acid clearance. Due to the glomerular filtration become impaired proteinuria develops. An increase in plasma urete is therefore an early sign in the evolution of preeclampsia [19]. In the present study the significantly higher levels of uric acid levels are observed in preeclamptic women when compared to controls. Vitamin C is an antioxidant which may be referred to as reducing agent. It reacts with superoxide and other lipid hydroperoxide radicals. This study observed plasma vitamin C level to be higher in women with apparently normal pregnancy than in those with pre-eclampsia [20].

In this study, preeclamptic women showed higher serum concentrations of total cholesterol, LDL, VLDL and TG when compared to the normal pregnant women which was statistically significant. In this study, a statistically significant decrease in HDL cholesterol was observed in preeclamptic women. This is in agreement with previous studies Sayyed A et al. [21] which have shown increased cholesterol, TG, LDL, VLDL levels in preeclamptic women when compared to controls Usha Adiga et al. [22] 
conducted study at Mangalore which showed increased in total cholesterol, decreased in HDL cholesterol levels.

\section{CONCLUSION}

The imbalance between oxidative damage and antioxidant defences in preeclampsia leads to endothelial cell dysfunction and it appears to be a central feature in the patho-physiology of pre-eclampsia. Endothelial cell dysfunction in glomerula leads to reduction in renal perfusion and glomerular filtration in preeclampsia. While this condition, developing of hyper uricemia by impairment of tubular function and reduction in uric acid clearance. Due to the glomerular filtration become impaired proteinuria develops. An increase in plasma urete is therefore an early sign in the evolution of preeclampsia. Vitamin $C$ is an antioxidant which may be referred to as reducing agent. It reacts with superoxide and other lipid hydroperoxide radicals. This study observed plasma vitamin Clevel to be higher in women with apparently normal pregnancy than in those with pre-eclampsia.

\section{REFERENCES}

[1]. Morris JM, Gopaul NK, Endresen MJ, Knight M, Linton EA, Dhir S, et al. Circulating markers of oxidative stress are raised in normal pregnancy and preeclampsia. $\mathrm{Br} J$ Obstet Gynaecol. 1998;105(11):1195-9.

[2]. Hubel CA. Oxidative Stress in the Pathogenesis of Preeclampsia. Proc. Soc. Exp. Biol. Med Review. 1999;222(3):222-35.

[3]. Working Group report on high blood pressure in pregnancy: Consensus report. Working Group on High Blood Pressure in Pregnancy. National High Blood Pressure Education Program. Am J Obstet.Gynecol. 1990;163:1689-712.

[4]. Halliwell B, Gutteridge JMC. Role of free radicals and catalytic metal ions in human disease; an overview. Methods in Enzymology 1990;186:1-85.

[5]. Nakai A, Oya A, Kobe H, Asakura H, Yokota A, Koshino $\mathrm{T}$, Araki T. Changes in maternal lipid peroxidation levels and antioxidant enzymatic activities before and after delivery. J Nippon Med Sch 2000;67(6):434-439.

[6]. Potter JM, Nestel PJ. The hyperlipidemia of pregnancy in normal and complicated pregnancies. AM J Obstet Gynecol 1979;133:165-70.

[7]. Walsh SC. Lipid peroxidation in pregnancy. Hypertension Pregnancy 1994;13:1-25.

[8]. Hubel CA, Roberts JM, Taylor RN, Musci TJ, Rogers GM, Mc Laughlin MK. Lipid peroxidation in pregnancy: New perspectives on preeclampsia. Am. J. Obstet. Gynecol. 1989;161:1025-34.
[9]. Dutta DC, Konar HL, Test Book of Obstetrics, 6t h edn. New Central Book Agency (P) Ltd. 2004;222-3.

[10]. Kharb S. Vitamin E and C in pre-eclampsia. Eur J Obstet Gynecol Reprod Biol 2000;93(1):37-9.

[11]. Dursen P, Demirtas E,Bayrak A, Haken Yarli. Decreased serum paraoxonase 1 (PON1) activity: an addi-tional risk factor for atherosclerotic heart disease in pa-tients with PCOS. Human Reproduction. 2006:21(1):104-8.

[12]. Lam C, Lim KH, Kang DH, Karumanchi SA. Uric acid and preeclampsia. Semin Nephrol.2005;25(1):5660.

[13]. Zhang C, Williams MA, King IB, Dashow EE, Sorensen TK, Frederick IO, Thompson ML, Luthy DA.Vitamin C and the risk of preeclampsia-results from dietary questionnaire and plasma assay. Epidemiology.2002:13(4):409-16.

[14].Kashinakunti SV, Sunitha H, Gurupadappa K, Shankarprasad DS. Lipid peroxidation and antioxidant status in Preeclampsia. Al Ameen J Med Sci.2010:3(1):38-41.

[15].Sharma JB, Sharma A, Bahadur A, Vimala N. Oxidative stress markers and antioxidant levels in normal pregnancy and preeclampsia. International journal of Gynecology and Obstretics. 2006:94;237.

[16].Bowen RS, Moodley J, Dutton MF, Theron AJ. Oxidative stress in pre-eclampsia. Acta Obstet Gynecol Scand. 2001;80(8):719-25.

[17]. Atiba AS, Abbiyesuku FM, Niran-atiba TA , Oparinde DP, Ajose OA, Akindele RA.Free radical attack on membrane lipid andAntioxidant vitamins in the course of preeclamptic Pregnancy. Ethiop J Health Sci. 2014;24:35-42.

[18]. Yoneyama Y, Sawa R, Suzuki S, Doi D, Yoneyama K, Otsubo $Y$, et al. Relationship between plasma malondialdehyde levels and adenosine deaminase activities in preeclampsia. Clin Chim Acta. 2002 322(12):169-73.

[19]. Kamath KR, Nayak S.R. Shantharam M. Serum Uric acid level in preeclampsia and its correlation to maternal and fetal outcome. IJBR . 2014;05(01):224.

[20].Sagen N, Haram K, Nilsen ST. Serum urate as a predictor of fetal outcome in severe pre-eclampsia. Acta Obstet Gynecol Scand. 1984;63(1):71-5.

[21]. Sayyed A, Sontakke A. Study of Lipid Peroxidation and Antioxidant Status in Preeclampsia. JKIMSU. 2013;2(2):70-76.

[22]. Adiga U, D'souza V, Kamath A, Mangalore N. Antioxidant activity and lipid peroxidation in preeclampsia. J Chin Med Assoc. 2007 Oct 70(10):4358.

How to cite this article: Anita Ramesh Annaldasula, $K$ Durgaprasad. DYSLIPIDEMIA AND ANTIOXIDATIVE SYSTEM IN PREECLAMPTIC WOMEN AT RURAL AREA OF RAYALASEMA. Int J Intg Med Sci 2017;4(6):517-521. DOI: 10.16965/ijims.2017.114 